\title{
Operation Optimal Control of Urban Rail Train Based on Multi-Objective Particle Swarm Optimization
}

\author{
Liang Jin ${ }^{1, *}$, Qinghui Meng ${ }^{1}$ and Shuang Liang ${ }^{2}$ \\ ${ }^{1}$ Department of Mechanical and Electrical, Henan Polytechnic Institute, Nanyang Henan, 473000, China \\ ${ }^{2}$ University of Florence, Firenze, 50041, Italy \\ *Corresponding Author: Liang Jin. Email: 19530521@163.com \\ Received: 09 February 2021; Accepted: 04 May 2021
}

\begin{abstract}
The energy consumption of train operation occupies a large proportion of the total consumption of railway transportation. In order to improve the operating energy utilization rate of trains, a multi-objective particle swarm optimization (MPSO) algorithm with energy consumption, punctuality and parking accuracy as the objective and safety as the constraint is built. To accelerate its the convergence process, the train operation progression is divided into several modes according to the train speed-distance curve. A human-computer interactive particle swarm optimization algorithm is proposed, which presents the optimized results after a certain number of iterations to the decision maker, and the satisfactory outcomes can be obtained after a limited number of adjustments. The multiobjective particle swarm optimization (MPSO) algorithm is used to optimize the train operation process. An algorithm based on the important relationship between the objective and the preference information of the given reference points is suggested to overcome the shortcomings of the existing algorithms. These methods significantly increase the computational complexity and convergence of the algorithm. An adaptive fuzzy logic system that can simultaneously utilize experience information and field data information is proposed to adjust the consequences of off-line optimization in real time, thereby eliminating the influence of uncertainty on train operation. After optimization and adjustment, the whole running time has been increased by $0.5 \mathrm{~s}$, the energy consumption has been reduced by $12 \%$, the parking accuracy has been increased by $8 \%$, and the comprehensive performance has been enhanced.
\end{abstract}

Keywords: Particle swarm optimization; multi-objective; urban rail train; optimal control

\section{Introduction}

The train operation process is a complex control issue involving safety, energy saving, punctuality, comfort, and accurate stop. We must seek a "satisfactory" strategy to optimize the whole operation process from a variety of operation modes to ensure the security, reliability and coziness of the train operation process, while energy consumption and operation time can reach the acceptable range of

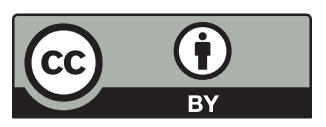

This work is licensed under a Creative Commons Attribution 4.0 International License, which permits unrestricted use, distribution, and reproduction in any medium, provided the original work is properly cited. 
railway operation department and passengers. In order to achieve this goal, global railway science and technology workers have conducted extensive and in-depth research. The study [1] assumed that the operation strategy consisted of a limited input control sequence, and the energy consumption of the train under each input control changes was constant over time. The optimal duration of each input control can be determined by an optimization method, so that the energy consumption of the train under this operation strategy can be minimized. In the paper [2], the train operation control was transformed into an optimization problem with energy consumption as the objective, and the train kinetic energy was used as an independent state variable. Thus, the optimal control strategy with the minimum train energy consumption can be obtained by the maximum principle. In addition, Liu et al. [3] indicated that the control strategy that minimizes the energy consumption of the train can be attained under the continuous and discrete control variables. However, this control strategy was achieved by a detailed discussion of maximum principle. Also, the optimal control strategy with the minimum train energy consumption was obtained via the empirical method and the maximum principle [4]. The train operation mode was analyzed, and the energy-saving strategies were put forward. Moreover, Ref. [5] showed that the operation section can be divided into several typical sections. When the train operated in the optimal energy consumption strategy mode, the train energy consumption and operation time in this section have a certain functional relationship. This paper indicates that the offline mode can be applied to acquire this functional relationship, and then the global optimization method can be employed to optimize the operation time. As shown in Ref. [6], the train operation process can be divided into five sub-processes: starting, steady acceleration, constant speed operation, speed regulation, and braking and parking. The fuzzy neural network method is used to get the operation strategy, so as to control the train.

Many of the above-mentioned research works have been successfully applied to engineering practice. However, most of these studies regarded the train operation process as a single objective optimization problem, and the result was only one of several feasible operation strategies. Sometimes, it may not be possible to obtain a current satisfactory solution in one operation, and multiple optimization operations were required. This did not reflect that the train operation process itself was a multi-objective problem. The studies [7,8] employed a multi-objective optimization method to investigate the train operation control, but the traditional weighted sum method to solve the multi-objective optimization. Due to the limitations of this method, it failed to reflect the characteristics of the multi-objective optimization. Also, Huang et al. [9] utilized the multi-objective optimization method to optimize the membership function parameters of the fuzzy predictive controller in urban rail transit, with the satisfactory results achieved.

In this paper, based on the control objectives of train energy consumption, running time and parking accuracy, the multi-objective optimization model is established with the constraints of the speed limit conditions and motion equations. The model is solved by the multi-objective particle swarm optimization algorithm (MOPSO). In one operation, multiple Pareto optimal solutions of the problem can be obtained. Each Pareto optimal solution corresponds to a set of feasible train operation control strategies, and each set of strategies can achieve a "balance" effect among various indexes of train energy consumption, operation time and parking accuracy. A set of appropriate control strategies can be selected according to the current operation condition of the train (punctual, early or late operation). If the train is controlled according to this strategy, satisfactory results can be obtained.

\section{Multi Objective Optimization Model of Train Operation Process}

There are many control strategies when the train is running within the given time in the operation diagram. Drivers and passengers can choose any operation mode to control the train according to the current running speed, time, and distance from the parking station. The prerequisite for reducing energy consumption is to ensure that the train stops on time and safely at an appropriate location on the platform and does not exceed the speed limit. However, the disadvantages of the control strategy increase the 
energy consumption of the train. Here, a group of actual operation data is quoted to explain the problem. In the Shibalihe section to the airport section of the Mengzhuang Locomotive Depot, the operation distance is $16 \mathrm{~km}$, and with the upward direction, 6-car formation B-type train, the passenger car will consume $4900 \mathrm{kw}$ $\cdot h$ and $5800 \mathrm{kw} \cdot \mathrm{h}$ corresponding to the good and poor operation methods. It can be seen that due to different operation strategies, the difference in power consumption of a single train accounts for the entire operation consumption. With $1 / 6$ of the electricity consumption, all maintenance departments of the whole railway are universal to operate the train at will. Therefore, it is of great practical and economic significance to optimize the train operation process with the goal of train energy consumption, operation time and parking accuracy.

In this paper, the motion equation of train particle is shown in Eq. (1) $[3,10]$ :

$$
\left\{\begin{array}{l}
\frac{d t}{d x}=\frac{1}{v} \\
v \frac{d v}{d x}=f(u, v)-\omega(x, v)-b(u, v)
\end{array}\right.
$$

In which: $t(0)=0, t(x)=t, V(0)=V(X)=0, V(t)<V$.

$x \in[0, X]$ is an independent variable that represents the position of the train on the line; $X$ is the total length of the line; $t \in[0, T]$ and $v \in[0, V]$.

$x$ is the state variable, and $\mathrm{t}$ is the running time, which is the specified whole running time. $V$ is the train running speed and is also the maximum allowed running speed, while $[t, v]=[0, T] \times[0, V] \subseteq R^{2} ; u \in\{-1,0$, $1\}=U$ is the input control sequence, where " -1 " is the brake, " 0 " is the coasting, and " 1 " is the traction; $f(u, v)$ is the traction force, determined by the locomotive (train) traction characteristic curve, which is related to the input control and the running speed; $\omega(x, v)$ is the additional resistance of the train that is determined by the position and running speed of the train on the line; $b(u, v)$ is the braking force, which is established by the input control, running speed and braking characteristics of the train. Typically, when the train is in the traction state, $u=1, f(u, v)>0, b(u, v)=0$; when the train is in the inert operation, $u=0$, $f(u, v)=0, b(u, v)=0$; when the train is in braking, $u=-1, f(u, v)=0$. All energy consumption on the train $(0, X]$ will be:

$J=\int_{0}^{X} \phi(u) d x$

where, $\phi U \rightarrow R$ is the energy consumption corresponding to the different input control of the train.

The operation time of the total line:

$$
\left\{\begin{array}{l}
T^{\prime}=\int_{0}^{X} t(x) d x \\
f_{t}=\left(1+\tau_{0}\right)^{\left(T^{\prime}-T\right) / \tau}
\end{array}\right.
$$

In Eq. (3) [11], $\tau_{0}$ is the coefficient of the penalty term, reflecting the unit energy consumption required to ensure the train on time when the delay time is within $\tau$ (the acceptable delay time limit); $f_{t}$ is the punctuality rate index. Within a certain early and late time limit $\tau$, the $f_{t}$ value is relatively trivial. When the train operation exceeds the early time limit, the value of $f_{t}$ will be also relatively small. When the delay time $\tau, f_{t}$ increases rapidly, so this value can reflect the punctuality of the train operation.

When the train stops, the actual parking place is:

$$
\left\{\begin{array}{l}
X^{\prime}=x\left(T^{\prime}\right), X^{\prime} \leq X \\
f_{d}=e^{\left|X^{\prime}-X\right| / 1000}
\end{array}\right.
$$


In Eq. (4), the value of $f_{d}$ gives the difference between the actual running distance $d$ of the train and the total length of the line, which also indicates the position of the train on the line after parking. The larger the deviation from the actual end point, the larger $f_{d}$. Therefore, the accuracy of the train stopping can be evaluated with $f_{d}$.

The problem of the multi-objective train optimal control is to find a set of $\left\{x_{i}\right\}, i=1,2, \ldots, k$. For the system described in Eqs. (1) and (2), $\left\{u_{i}\right\}, i=1,2, \ldots, k$ makes the energy consumption $J$, the punctuality rate index $f_{t}$ and the parking accuracy index $f_{d}$ of the train minimum, where $u_{i} \in U, x_{i} \in[0, X], i=1,2, \ldots, k, k$ is unknown.

With $J, f_{t}$ and $f_{d}$ as the objectives, the multi-objective optimization problem is considered:

$$
\left\{\begin{array}{l}
\min J, f_{t}, f_{d} \\
\text { s.t. } \frac{d t}{d x}=\frac{1}{v} \\
\text { s.t. } \frac{d v}{d x}=f(u, v)-\omega(x, v)-b(u, v) \\
t(0) \stackrel{=}{=}, t(X)=T, v(0)=v(X)=0, v(t)<V
\end{array}\right.
$$

\section{A Multi-Objective Particle Swarm Optimization Algorithm for Train Operation}

From the above analysis, it can be seen that the optimization of train operation process needs to determine a set of train input control sequences $\left\{u_{1}, u_{2}, \ldots, u_{k}\right\}, u_{i} \in\{-1,0,1\}, i=1,2, \ldots, k$, and the conversion point of each input control sequences $\left\{x_{i}\right\}, i=1,2, \ldots, k$, that is, the distance of train operation under each input control sequence $\left\{d_{i}\right\}, i=1,2, \ldots, k$.

Where, $d_{1}=x_{1}, d_{j}=x_{j}-x_{j-1}, j=1,2, \ldots, k$.

In order to determine the initial input control sequence, according to the requirements of the train traction calculation regulations, the input control sequence of the calculation results shall be optimized by the following strategies: it ignores the control sequence with the running distance less than the minimum $d_{i}$ value to ensure the coasting between traction and braking [12-14]. Many researches are committed to solving the multi-objective optimization problem by the particle swarm optimization algorithm. Following the emergence of many multi-objective particle swarm optimization algorithms, engineering applications have attracted more and more attention. In this paper, the improved MOPSO is applied to train the input control sequence operation distance $\left\{d_{i}\right\}, i=1,2, \ldots, k$, which is optimized to obtain the satisfactory control effect.

The improvement of MOPSO is mainly reflected in the following two aspects:

(1) Under certain conditions, the traditional grid method can guarantee.

The solution converges to the real Pareto optimal solution with probability 1, and can keep the diversity of the solution, and the computational time complexity is better than the niche method, so it has been widely used. By dividing the target space into a series of limited subspaces, the optimality and diversity are respectively considered in these subspaces. However, this contradiction exists because the target space cannot be understood in advance or even infinitely. The reason for this problem is that the multi-objective optimization algorithm with grid to keep the diversity of solutions does not meet the convergence condition. The convergence of grid method is conditional, which requires the upper bound of the grid to converge. That is to say, the Pareto front can traverse the whole target space. Except for the two-objective optimization, most of the multi-objective optimization have no problem to satisfy this condition.

In order to improve the adverse effect on the solution, a nonlinear tangent function is used to map the objective value of all solutions to a limited range [11-14], 
$F\left(w_{i}\right)=\tan \left(\left(w_{i}-w_{\min i}\right) s_{i}\right)$

where: $w_{i}$ is the value of target I of any solution in the target space, $w_{\max i}$ and $w_{\min i}$ are the maximum and minimum values of target I, respectively, where $i=1,2,3 . s_{i}=\arctan \left(\pi / 2-\epsilon_{i}\right) /\left(w_{\max i}-w_{\min i}\right)$, and $\varepsilon_{i}$ is not greater than $\pi / 4$. It can be seen that $w_{\max } i$ and $+\infty$ are correspondingly mapped to $\pi / 2-\varepsilon$ and $\pi / 2$. Consequently, when dividing the target space, the contradiction between the fineness of each sub region and the infinite possibility of the whole target space is eliminated, and the upper bound of the adaptive grid is guaranteed to be bounded, thereby improving the convergence of the algorithm.

(2) Using the improved e-dominance method to determine the winning relationship between the solution vectors.

The research shows that the above measures can significantly improve the convergence and computational complexity of MOPSO. Compared with other MOPSO applications, they are different in the following aspects:

(1) In order to make the particle swarm as close to the target area as possible, this paper adopts the following method: $X$ is the total length of the line, and the maximum allowable operating speed $V,\left\{d_{i}\right\}, i=1,2, \ldots, k$, is the distance of the train running in the control sequence $i$, which is the optimization object. Since the train must maintain a certain time (take $3 \mathrm{~S}$ ) in each input control state, $d_{i}$ has a minimum value, which can be calculated in $v$. Also, the initialization range of $d_{i}$ is as follows:

$d_{i}:[3 V, X]$ Di: $[3 \mathrm{~V}, \mathrm{x}]$, the unit of $V$ is $\mathrm{m} / \mathrm{s}$, and the unit of $X$ is $\mathrm{m}$, di

$d_{i}:\left[3 V, X-\sum_{k=1}^{i} d_{k}\right], d_{i}>0, i=1,2, \ldots, k$.

(2) Best selection strategy: The improved grid can be taken as the diversity keeping strategy, because the basis of all control strategies for comparison is that the train runs in the same section to ensure the safety of the train. Therefore, the basic requirement of all non-inferior solutions is that $\sum_{k=1}^{i} d_{k}$ should be as close to $X$ as possible, and the solution of $\sum_{k=1}^{i} d_{k}$ closest to $X$ in the grid with the least congestion should be selected as best.

(3) Adopt the elite retention strategy of external population archives. When the number of non inferior solutions exceeds the capacity of archives, it can start pruning operation according to the following strategies: First, those solutions of the overspeed can be eliminated in the operation process to ensure the safety of the train. Second, those solutions can be discarded for the farthest from the actual operation distance x. Finally, a delete can be randomly selected from the grid with the largest density until the file capacity is met.

The multi-objective optimization algorithm steps of the train operation process are as follows:

(1) Initialize the line parameters, line speed limit, train basic parameters, inertia coefficients and learning factors of the particle swarm optimization algorithm;

(2) Run traction calculation subroutine;

(3) Optimize the input control sequence in the traction calculation results according to the following methods: the input can be ignored with the running distance less than the minimum value of $\mathrm{Di}$ to ensure existing the coasting between traction and braking;

(4) Run the particle swarm optimization subprogram; 
(5) The optimal control strategy of the completed train. The solution can be selected according to the following methods:

A) When running on time, it can be selected the solution for the on-time rate index $\mathrm{ft}$ greater than 1 but closest to 1 ;

B) When running early, the solution can be chosen with the punctuality index $\mathrm{ft}$ greater than 1 , and the actual running time can be controlled with the strategy composed of the solution.

The difference greater than the specified operation time is equal to (or closest to) the earlier time;

C) When the train is running late, the solution can be picked with the punctuality index $\mathrm{ft}$ less than 1 , and the actual running time can be regulated with the strategy composed of the solution.

The difference less than the specified operation time is equal to (or closest to) the time of delay.

\section{Simulation Experiment Results and Analysis}

Based on a-16 km-long section of passenger dedicated line of Line 2, a high-speed train is simulated.

The running time of the train interval is $2160 \mathrm{~s}$, and the early and late time is $30 \mathrm{~s}$. The particle swarm size of the MOPSO algorithm is 80 , the external population file is 30 , and the number of iterations is 200 . The simulation experiment is carried out in MATLAB 7.0.

Firstly, the train operation speed distance curve in Fig. 1 and control strategy are attained from the traction calculation procedure, as shown in Figs. 2 and 3. In the figure, the input control sequences 1, 0 and - 1 represent traction, coasting, and braking, respectively. The train tracks the limited speed with the maximum capacity all the time, the minimum operation time can be obtained with $1800 \mathrm{~s}$, but the energy consumption is very large, the energy consumption index parameter is 911 . 1 , the train condition changes frequently, and the long time is close to the limit speed operation. Also, there are security risks, and the train operation security is reduced, such a control strategy is not suitable for the train control [15-19].

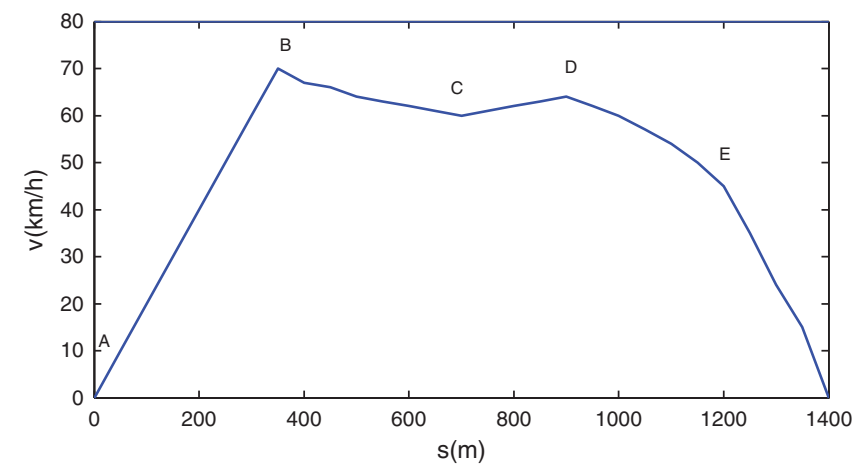

Figure 1: The velocity-distance (V-S) curve and transition points

Through analysis and optimization, the "traction coasting traction coasting braking coasting braking" is determined as the input control sequence, and the multi-objective particle swarm optimization algorithm introduced in this paper is used to optimize the conversion points of the control sequence. 


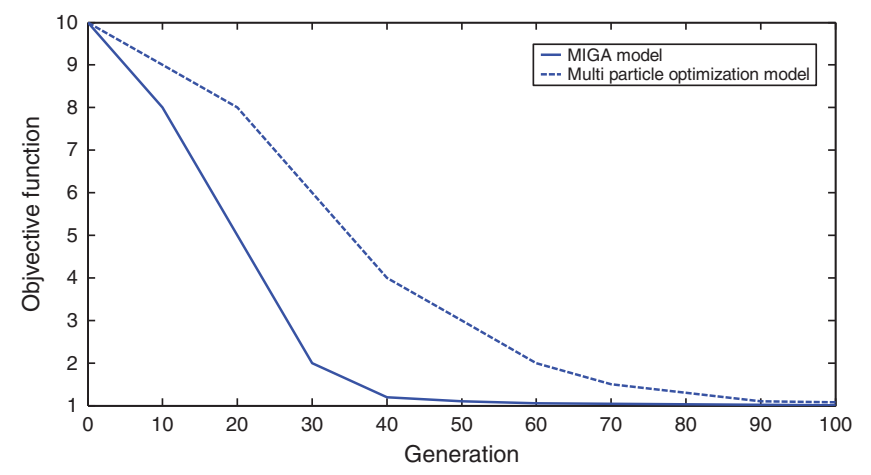

Figure 2: The cure between generation and objective function

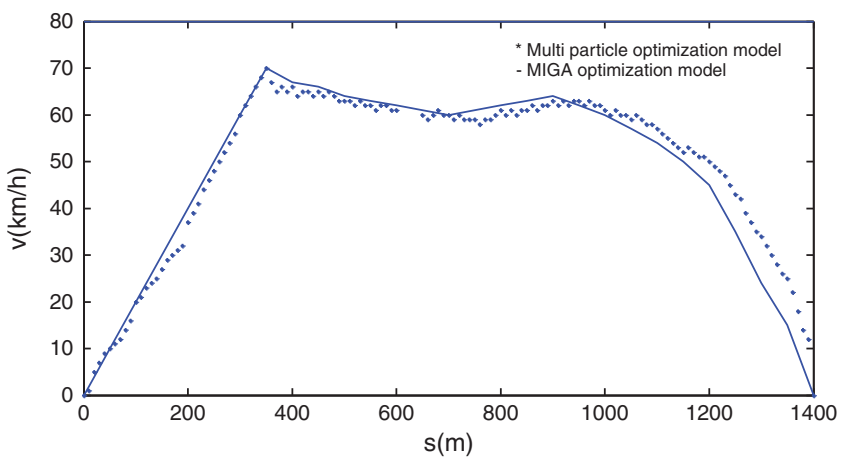

Figure 3: Train operation control curves of multi particle optimization model and MIGA

The multi-objective particle swarm optimization algorithm can achieve the multiple optimal solutions in one operation process, so it can get the multiple train control strategies. The train control by these strategies has certain flexibility, with obtaining the satisfactory results under the corresponding circumstances. The energy consumption of the train is relatively small when the interval operation time is fully utilized. If the train is running on time, the operation can be realized. The time is only $5 \mathrm{~s}$ longer than the specified time, the energy consumption is relatively low, and the error of stop position is also relatively small, because there is always a certain safety distance between the train and the actual protection site. It can be seen that the optimized control strategy does not make the train track and limit the speed operation in the whole section, but also maintains a relatively high average speed. If the train is running late, the strategy can make the train $61 \mathrm{~s}$ late arrive at the terminal on time. Otherwise, if the train is running early, the control strategy with slightly longer running time can be selected, which can make full use of the earlier time and reduce energy consumption [20-25].

\section{Conclusions}

The train operation process is a complex multi-objective optimization problem. There are certain contradictions and incommensurability between the train safety, energy consumption, operation time, parking accuracy and comfort, which increase the difficulty of problem solving. The development of multi-objective optimization theory adds vitality to the solution of the similar issues, especially the multiobjective evolutionary algorithm and multi-objective particle swarm optimization algorithm. To solve the problem of train optimal control, people have paid extensive attention to improving the multi-objective particle swarm optimization method. The method to keep the diversity of solutions is studied, with the corresponding strategies proposed. Moreover, this work gives the algorithm of the multi-objective particle 
swarm optimization algorithm to optimize the train operation process. A large number of simulation studies also have verified the effectiveness and feasibility of the strategies and algorithms.

Acknowledgement: Thank for the hard work of the team members, the scientific research platform provided by the university and the strong support of government funds.

Funding Statement: This work was supported by the project of science and technology of Henan province under Grant No. 202102210134.

Conflicts of Interest: The authors declare that they have no conflicts of interest to report regarding the present study.

\section{References}

[1] Y. Zhang, J. Dang, X. Liu and Y. Fan, "Optimized handover algorithm based on stackelberg games in CBTC systems for urban rail transit," Journal of Engineering Science \& Technology Review, vol. 7, no. 3, pp. 127-132, 2014.

[2] K. X. Wen, Z. Peng and Q. N. Li, "Last train delay management in urban rail transit network: Bi-objective MIP model and genetic algorithm," Ksce Journal of Civil Engineering, vol. 22, no. 3, pp. 1436-1445, 2018.

[3] F. Liu, J. Xun, and N. Bin, "An optimization method for train driving trajectory in urban rail systems, Chinese association of automation," in IEEE, Wu Han, WH, China, pp. 413-418, 2017.

[4] J. Su, R. Xu, S. Yu, B. Wang and J. Wang, "Idle slots skipped mechanism based tag identification algorithm with enhanced collision detection," KSII Transactions on Internet and Information Systems, vol. 14, no. 5, pp. 22942309, 2020.

[5] J. Su, R. Xu, S. Yu, B. Wang and J. Wang,"Redundant rule detection for software-defined networking," KSII Transactions on Internet and Information Systems, vol. 14, no. 6, pp. 2735-2751, 2020.

[6] P. Wang, R. M. P. Goverde and L. Ma, "Multiple-phase train trajectory optimization method under real-time rail traffic mmanagement," in Int. Conf. on Intelligent Transportation Systems. IEEE, Las Palmas, Spain, pp. 1214412152, 2015.

[7] P. L. Wang, R. M. P. Goverde and F. Mannering, "Multi-train trajectory optimization for energy efficiency and delay recovery on single-track railway lines," Transportation Research Part B Methodological, vol. 105, no. 4, pp. 340-361, 2017.

[8] H. Shi, Q. Peng and H. Guo, "Traction calculation model of urban mass transit," Journal of Traffic and Transportation Engineering, vol. 5, no. 4, pp. 20-26, 2005.

[9] Y. Huang, X. Ma and S. Su, "Optimization of train operation in multiple interstations with multi-population genetic algorithm," Energies, vol. 8, no. 12, pp. 14311-14329, 2005.

[10] W. K. Xu, P. Zhao and L. Q. Ning, "A passenger-oriented model for train rescheduling on an urban rail transit line considering train capacity constraint," Mathematical Problems in Engineering, vol. 2017, no. 4, pp. 1-9, 2017.

[11] C. Lin, X. Q. Fang and X. Zhao, "Study on energy-saving optimization of train coasting control based on multipopulation genetic algorithm," in Int. Conf. on Control, Automation and Robotics. IEEE, Nagoya, NGY, Japan, pp. 627-632, 2017.

[12] R. Su, Q. Gu and T. Wen, "Optimization of high-speed train control strategy for traction energy saving using an improved genetic algorithm,” Journal of Applied Mathematics, vol. 2014, no. 2, pp. 1-7, 2014.

[13] Y. Song and W. Song, "A novel dual speed-curve optimization based approach for energy-saving operation of high-speed trains," IEEE Transactions on Intelligent Transportation Systems, vol. 17, no. 6, pp. 1564-1575, 2016.

[14] J. Zhu and B. Zhou, "Optimization design of rc ribbed floor system using eagle strategy with particle swarm optimization," Computers, Materials \& Continua, vol. 62, no. 1, pp. 365-383, 2020.

[15] J. Su, Z. Sheng, A. X. Liu and Y. Chen, "A partitioning approach to RFID identification," IEEE/ACM Transactions on Networking, vol. 28, no. 5, pp. 2160-2173, 2020.

[16] J. Su, Z. Sheng, A. X. Liuand Y. Chen, "Captureaware identification of mobile RFID tags with unreliable channels," IEEE Transactions on Mobile Computing, vol. 56, no. 12, pp. 1-14, 2020. 
[17] Z. Zhu, X. Guo and J. Zeng, "Route design model of feeder bus service for urban rail transit stations, Mathematical Problems in Engineering, vol. 2017, no. 2, pp. 1-6, 2017.

[18] Y. Liu, X. Yan, F. Yan, Z. Xu and W. Shang, "Sliding-mode PID control of UAV based on particle swarm parameter tuning," Computers, Materials \& Continua, vol. 63, no. 1, pp. 469-487, 2020.

[19] Y. Huang, L. Yang and T. Tang, "Saving energy and improving service quality: Bicriteria train scheduling in urban rail transit systems," IEEE Transactions on Intelligent Transportation Systems, vol. 17, no. 12, pp. 1-16, 2016.

[20] J. T. Yin, T. Tangand B. Ran, "Energy-efficient metro train rescheduling with uncertain time-variant passenger demands: An approximate dynamic programming approach," Transportation Research Part B, vol. 91, no. 53, pp. 178-210, 2016.

[21] A. M. Farhan, "Effect of rotation on the propagation of waves in hollow poroelastic circular cylinder with magnetic field," Computers, Materials \& Continua, vol. 53, no. 2, pp. 129-156, 2017.

[22] N. Mohanapriya and D. B. Kalaavathi, "Adaptive image enhancement using hybrid particle swarm optimization and watershed segmentation," Intelligent Automation \& Soft Computing, vol. 25, no. 4, pp. 663-672, 2019.

[23] X. Chen and J. H. Jiang, "A method of virtual machine placement for fault-tolerant cloud applications," Intelligent Automation \& Soft Computing, vol. 22, no. 4, pp. 587-597, 2016.

[24] X. F. Li, Y. B. Zhuang and S. X. Yang, "Cloud computing for big data processing," Intelligent Automation \& Soft Computing, vol. 23, no. 4, pp. 545-546, 2017.

[25] K. Vijayalakshmi and P. Anandan, "Global levy flight of cuckoo search with particle swarm optimization for effective cluster head selection in wireless sensor network," Intelligent Automation \& Soft Computing, vol. 26, no. 2, pp. 303-311, 2020. 